\title{
Diversidad de Araneae en cultivos de Citrus sinensis (Rutaceae) de la Provincia de Corrientes, Argentina
}

\author{
Gilberto Avalos ${ }^{1}$, María Esther Bar ${ }^{1}$, Elena Beatriz Oscherov ${ }^{1} \&$ Alda González ${ }^{2}$ \\ 1. Cátedra de Biología de los Artrópodos, Facultad de Ciencias Exactas y Naturales, Universidad Nacional del Nordeste, \\ Avda. Libertad 5470 (3400) Corrientes, Argentina; gilbertoa@exa.unne.edu.ar, mariaestherbar@yahoo.com.ar, \\ ebosche@exa.unne.edu.ar \\ 2. Centro de Estudios Parasitológicos y de Vectores (CEPAVE) (CONICET LA PLATA-UNLP), Calle 2 NN$^{\circ} 584$ (1900) \\ La Plata, Argentina; asgonzalez@cepave.edu.ar
}

\author{
Recibido 07-VI-2012. Corregido 22-I-2013. Aceptado 19-II-2013.
}

\begin{abstract}
Spider diversity in cultures of Citrus sinensis (Rutaceae) in Corrientes province, Argentina. Spiders are predators that reduce insect pest populations in agroecosystems. In spite that the presence of spider assemblages has been described in different crop plants, no reports have been done for Citrus species in Argentina. We studied the spider community associated with cultures of Citrus sinensis in the province of Corrientes, Argentina, in two plots (AM1 irrigated and AM2 unirrigated) of 0.82 hectares each. Spiders were collected monthly by using pitfall traps, hand capture, agitation of foliage and sieving of soil litter. A total of 7174 specimens were collected (33 families, 44 genera and 200 species/morphospecies). The AM1 recorded 3811 individuals (33 families, 179 species/morphospecies) and AM2 3363 individuals (31 families, 174 species/morphospecies). November, December and January showed the highest abundance in both plots. A total of $70 \%$ of collected spiders were juveniles. Eight families were the most abundant and represented $75 \%$ of spiders collected in both plots, the largest being Lycosidae. We identified eight guilds in both plots, for which the ground runners resulted the most abundant (AM1 $n=1341, s=39, n=999$ AM2, s=33), followed by orb weavers (AM1 n=637, s=36; AM2 n=552, s=33), the stalkers (AM1 n=471, s=43, AM2 n=453, s=47) and the space webbuilders (AM1 n=446, s=23; AM2 $\mathrm{n}=342, \mathrm{~s}=25$ ). The analysis of alpha diversity in both plots (AM1H'=4.161, $\mathrm{J}^{\prime}=0.802$; AM2 $\left.\mathrm{H}^{\prime}=4.184, \mathrm{~J}=0.811\right)$ showed no significant differences $(\mathrm{t}=1.083 \mathrm{p}=0.279)$. The linear dependences model was the one with the best fit results, where the species observed were more than estimated. Clench model estimated $90.9 \%$ of the spiders observed in the unit with irrigation and $90.6 \%$ in the unit without irrigation. Rev. Biol. Trop. 61 (3): 1243-1260. Epub 2013 September 01.
\end{abstract}

Key words: Araneae, diversity, agroecosystems, natural enemies, Citrus.

El manejo integrado de plagas (MIP) es un método ecológico que tiene como objetivo reducir o eliminar el uso de pesticidas y minimizar el impacto al medio ambiente (SAGPyA 2011). El MIP incluye como alternativa al control biológico, mediante el cual no se tiende a la erradicación de las plagas, sino a reducir la densidad de las mismas para impedir que causen daño económico. Es sabido que el éxito de estos programas dependerá de estudios preliminares sobre la biología y ecología del complejo de enemigos naturales presentes en el cultivo (Bale et al. 2008).
Entre los enemigos naturales depredadores se incluyen a las arañas con más de 40000 especies descritas a nivel mundial (Platnick 2012). Es notable su abundancia en los ecosistemas terrestres, tanto en ambientes naturales como agrícolas y ocupan un lugar importante entre los invertebrados depredadores más numerosos (Wise 1993). Existen evidencias experimentales que demuestran que las arañas tienen un valor potencial considerable desde el punto de vista del control biológico (Nentwig 1986, Riechert \& Lockley 1984, Young \& Edwards 1990, Nyffeler et al. 1992, 1994 
Sunderland 1999, Greenstone 1999, Benamú \& Aguilar 2001, Symondson et al. 2002, Liljesthrom et al. 2002, Maloney et al. 2003, Benamú 2004, Rodriguez et al. 2008, Armendano \& González 2010, 2011). Además de su capacidad para colonizar diferentes agroecosistemas, poseen adaptaciones que les permiten sobrevivir periodos largos de inanición, es decir cuando decrece notablemente la población de presas potenciales, no se dispersan como otros grupos de artrópodos depredadores (Greenstone \& Bennett 1980, Jackson 2000).

Se conocen diversos antecedentes sobre estudios de ensambles de arañas que habitan en sistemas agrícolas de plantas anuales y perennes, entre los que se pueden citar los efectuados en cultivos de manzano de Australia (Dondale 1966), de algodón de Texas (Dean \& Sterling 1987, Breene et al. 1993), de soja, trigo, algodón y alfalfa de Argentina (Liljesthrom et al. 2002, Beltramo et al. 2006, Armendano \& González 2010, 2011, Almada et al. 2012) y de cítricos de Australia, Israel, Uruguay, Perú y Costa Rica (Mansour et al. 1982, Mansour \& Whitcomb 1986, Benamú 1999, 2004, Elizondo Solís 2002).

La estructura del hábitat es importante al momento de considerar las distintas asociaciones de arañas en un agroecosistema, y determina la composición de los gremios de arañas que son grupos funcionales que pueden ejercer un control sobre diversos insectos plaga, pudiendo depredar sobre las distintas fases del ciclo biológico de una plaga, y a diferentes niveles dentro de un cultivo, sobre la copa, en el suelo, en la vegetación adyacente, o en la cubierta vegetal propia. Las especies que constituyen un grupo funcional presentan mejores habilidades competidoras que otras especies y la estructura en grupos funcionales constituiría una de las bases de la organización de una comunidad aracnológica (Uetz et al. 1999).

En la Provincia de Corrientes la producción citrícola abarca 25000 ha aproximadamente, de las cuales 9000 están habilitadas para la exportación a la Unión Europea, destino que demanda la máxima calidad y requisitos sanitarios para su ingreso. La fruta cítrica exportada a la UE y a otros mercados con similares restricciones debe cumplir, desde el origen, con altos estándares de calidad. Para esto SENASA (Servicio Nacional de Sanidad y Calidad Agroalimentaria) exige la aplicación de Buenas Prácticas Agrícolas (BPA) que implica la reducción del uso de agroquímicos, para garantizar la inocuidad y seguridad en la producción primaria (Ministerio de Producción, Trabajo y Turismo de Corrientes 2011).

El objetivo de este trabajo fue conocer la comunidad de Araneae en cultivos de Citrus sinensis (L) Osbeck (variedad Valencia Late), desarrollado bajo BPA y evaluar si difiere según el régimen de riego aplicado a las plantaciones. En la Argentina aún no se han realizado estudios referidos a la comunidad de arañas en cultivos de cítricos, por lo que el presente estudio representa la primera contribución al conocimiento de la diversidad, riqueza, abundancia y estructura de sus gremios en este tipo de cultivos.

\section{MATERIALES Y MÉTODOS}

La investigación se llevó cabo en cultivos de naranjos, ubicados en la Estación experimental del INTA Bella Vista localizada en la Cuenca Bellavistense, Provincia de Corrientes, Argentina, $\left(28^{\circ} 26^{\prime}\right.$ S-58 $\left.8^{\circ} 55^{\prime} \mathrm{W}\right)$. Este Departamento se halla a $70 \mathrm{~m}$ sobre el nivel del mar, el suelo es franco-arenoso, y el régimen pluviométrico es de $1200 \mathrm{~mm}$ anuales. La temperatura máxima absoluta es de $33^{\circ} \mathrm{C}$, la mínima absoluta de $8.5^{\circ} \mathrm{C}$ y la media es $20.5^{\circ} \mathrm{C}$ (Giménez et al. 2001). El área de estudio pertenece al Distrito Oriental Húmedo de la Provincia Fitogeográfica Chaqueña (Cabrera \& Willink 1973), Subregión Chaqueña, Provincia Chaco (Morrone 2001).

Los trabajos se realizaron durante un año, desde octubre 2005 a septiembre 2006, en dos parcelas de cultivos de Citrus sinensis de 0.82 ha y 392 plantas cada una. La parcela de muestreo uno (AM1) comprendió un cultivo con manejo de riego que recibe fertilizantes frecuentemente, y la parcela de muestreo dos 
(AM2) es un cultivo sin agua adicional y con el agregado de escasos fertilizantes.

Todos los meses y en cada parcela de muestreo se seleccionaron diez plantas de naranjo en base a un muestreo sistemático. Teniendo en cuenta el número total de plantas por área de estudio ( $\mathrm{n}=392$ ), cada mes se obtuvo un número aleatorio entre 0 y 1 , el cual se multiplicó por la longitud de la parcela $(119 \mathrm{~m})$, dando por resultado un número al azar; éste número se utilizó para seleccionar la planta a partir de la cual comenzó el muestreo. Desde la primera, se contabilizaron 39 plantas para obtener la segunda $(392 / 10=39.2)$ y así sucesivamente hasta llegar al árbol número diez, siguiendo una trayectoria de derecha a izquierda.

Para la recolección de arañas en la parte aérea del árbol, se utilizó la técnica de agitación de follaje y en el tallo captura manual. Para la recolecta de la araneofauna epigea se instalaron trampas de caída y se hicieron tamizados de hojarasca. De las cuatro técnicas utilizadas, se realizaron tres réplicas por cada árbol, totalizando doce muestras por árbol de muestreo.

La agitación del follaje consistió en sacudir vigorosamente un sector de la copa de la planta al azar sobre un paraguas entomológico de $0.70 \mathrm{~cm}$ de largo por $0.60 \mathrm{~cm}$ de ancho. $\mathrm{La}$ obtención de muestras tanto del follaje como del tallo se realizó durante $5 \mathrm{~min}$ para cada réplica. Se instalaron tres trampas de caída en la parte basal de cada árbol, formando un triángulo alrededor de él, a una distancia de $1.50 \mathrm{~m}$. Se utilizaron recipientes plásticos de $900 \mathrm{ml}$ con una abertura de $11 \mathrm{~cm}$ de diámetro. En cada uno de ellos se colocó etilenglicol diluido en agua en proporción 1:10 con gotas de detergente y se enterraron a nivel del suelo sin cubierta y permanecieron activas durante $48 \mathrm{hr}$. Se tamizaron superficies de $0.50 \mathrm{~m}^{2}$ de hojarasca sobre un lienzo blanco de $3 \mathrm{~m}^{2}$ con un tamiz de 10x15mm de apertura de malla, en tres lugares cercanos a cada árbol seleccionado.

En cada cultivo se obtuvieron 120 muestras por mes, totalizando 1440 muestras en el año por cada parcela muestreada.

Para la determinación de especies se utilizaron claves y descripciones específicas
(Birabén 1954, Bonaldo \& Brescovit 1992, Brescovit 1999, 1998, 1996, Brescovit et al. 2004, Corronca 1998, Gerschman de Pikelin \& Schiapelli 1965, Glueck 1994, Grismado 2001, Grismado \& Ramírez 2002, Grismado et al. 2009, Griswold et al. 2005, Höfer \& Brescovit 2000, Jocqué 1991, Levi 1977, 1980, 1985, 1988, 1991, 1991, 1992, 1993, 1995, 1996, 1997, 1999, 2001, 2004, Mello Leitâo 1929, Ramírez 2003, Rheims \& Brescovit 2001, Rheims \& Brescovit 2004, Traw 1995). Los individuos no identificados por especies se agruparon en morfoespecies, en base a caracteres estructurales diferenciables (Krell 2004).

Las arañas fueron separadas por estado del desarrollo y sexo. Las familias fueron agrupadas en gremios, diferenciándose principalmente con relación a la estrategia de forrajeo y/o a la conducta adoptada por el depredador al cazar su presa según Uetz et al. (1999) y Flórez (1999).

La riqueza específica (S) y diversidad específica total de cada área muestral (AM1 y AM2), se calculó con los índices de ShannonWienner $\left(H^{\prime}=\sum p_{i} \ln p_{i}\right)$ en base exponencial, el índice de dominancia de Simpson $\left(\lambda=\sum \mathrm{p}_{\mathrm{i}}{ }^{2}\right)$ y la equitatibilidad de Pielou $\left(\mathrm{J}^{\prime}=\mathrm{H}^{\prime} / \mathrm{H}^{\prime}{ }_{\text {max }}\right)$ (Moreno 2001). La significación estadística de estos índices se probó mediante pruebas de permutaciones (Good 2000) a un nivel de significación $\alpha=0.05$.

La estimación de la riqueza específica de las áreas, según datos de incidencia o de abundancia, se efectuó mediante estimadores de Chao $1 \quad\left(\mathrm{~S}_{\mathrm{Chao1}}=\mathrm{S}_{\text {obs }}+\mathrm{a}^{2} / 2 \mathrm{~b}\right)$, Chao $2\left(\mathrm{~S}_{\mathrm{Chao} 2}=\mathrm{S}_{\mathrm{obs}}+\mathrm{L}^{2} / 2 \mathrm{M}\right)$, Jackknife de primer orden $\left(\mathrm{S}_{\text {Jack } 1}=\mathrm{S}_{\text {obs }}+\mathrm{L}(\mathrm{n}-1 / \mathrm{n})\right.$ y Jackknife de segundo orden $\left(\mathrm{S}_{\mathrm{Jack} 2}=\mathrm{S}_{\mathrm{obs}}+[\mathrm{L}(2 \mathrm{n}-3) / \mathrm{n}-\mathrm{M}(\mathrm{n}\right.$ $\left.2)^{2} / \mathrm{n}(\mathrm{n}-1)\right)$, como también la curva de acumulación de especies (Morrone 2001), utilizando el programa EstimateS (Colwell 2010). Se representó, a través de dendrogramas, la similitud faunística entre los meses y área de muestro. Los análisis se realizaron con los programas estadísticos Biodiversity Pro (McAleece et al. 1997) y PAST (Hammer et al. 2001). 


\section{RESULTADOS}

Durante el año de muestreo se recolectaron en total 7174 arañas. Se identificaron 33 familias, 44 géneros y 200 especies/morfoespecies, de las cuales 163 estaban presentes en ambas parcelas, 26 estaban solamente en la AM1 y 21 en la AM2 (Cuadro 1).

En el AM1 se capturaron 3811 ejemplares identificados en 33 familias y 179 especies/ morfoespecies, de las cuales 27 (15.1\%) están representadas por un solo individuo (singletons) y $16(9 \%)$ con dos (doubletons). En el
AM2 se recolectaron 3363 individuos pertenecientes a 31 familias y 174 especies/morfoespecies, de las cuales 34 (19.5\%) correspondieron a singletons y $18(10.3 \%)$ a doubletons.

El $15.0 \%$ de las arañas recolectadas en la AM1 fueron adultos machos ( $\mathrm{n}=570)$, el 14.1\% adultos hembras $(\mathrm{n}=537)$ y el $70.9 \%$ pertenecieron a juveniles $(n=2704)$. En la AM2 el 14.9\% fueron adultos machos ( $\mathrm{n}=501)$, el 15.6\% adultos hembras $(n=525)$ y el $69.49 \%$ fueron juveniles $(n=2337)$. En la figura 1 se detalla la distribución mensual de las dos parcelas.

\section{CUADRO 1}

Especies y morfoespecies de arañas en cultivos de Citrus sinensis en Bella Vista, Corrientes, Argentina

TABLE 1

Species and morphospecies of spiders in cultures of Citrus sinensis in Bella Vista, Corrientes, Argentina

\begin{tabular}{|c|c|c|c|c|}
\hline Familia & Especies / Morfoespecies & AM1 & AM2 & Total \\
\hline Amphinectidae & Metaltella simoni Keyserling, 1878) & 31 & 65 & 96 \\
\hline Anyphaenidae & Arachosia praesignis (Keyserling, 1891) & 6 & 8 & 14 \\
\hline$”$ & Jessica erythrostoma (Mello-Leitão, 1939) & 143 & 135 & 278 \\
\hline$"$ & Jessica fidelis (Mello-Leitão, 1922) & 19 & 20 & 39 \\
\hline ” & Aysha triunfo Brescovit, 1992 & 13 & 12 & 25 \\
\hline$"$ & Jesica sp. 1 & 1 & 1 & 2 \\
\hline$"$ & Osoriella tahela Brescovit, 1998 & 7 & 1 & 8 \\
\hline Araneidae & Acacesia hamata (Hentz, 1847) & & 1 & 1 \\
\hline$"$ & Acacesia sp. 1 & 3 & & 3 \\
\hline$"$ & Aculepeira sp. 1 & & 1 & 1 \\
\hline$"$ & Alpaida bicornuta (Taczanowski, 1878) & 81 & 47 & 128 \\
\hline$"$ & Alpaida veniliae (Keyserling, 1865) & & 2 & 2 \\
\hline$"$ & Araneus uniformis (Keyserling, 1879) & 140 & 116 & 256 \\
\hline$"$ & Araneus sp. 1 & 37 & 22 & 59 \\
\hline$"$ & Araneus sp. 2 & 10 & 4 & 14 \\
\hline$"$ & Araneus sp. 3 & 6 & 17 & 23 \\
\hline$"$ & Araneus sp. 4 & 2 & 2 & 4 \\
\hline$"$ & Argiope argentata (Fabricius, 1775) & 1 & & 1 \\
\hline$"$ & Carepalxis sp. 1 & & 4 & 4 \\
\hline$"$ & Chorizopes sp. 1 & & 1 & 1 \\
\hline$"$ & Cyclosa diversa (O. P.-Cambridge, 1894) & 2 & & 2 \\
\hline$"$ & Cyclosa fililineata Hingston, 1932 & 1 & & 1 \\
\hline$"$ & Cyclosa serena Levi, 1999 & 1 & 1 & 2 \\
\hline$"$ & Cyclosa tapetifaciens Hingston, 1932 & & 3 & 3 \\
\hline$"$ & Cyclosa sp. 1 & 4 & 10 & 14 \\
\hline$"$ & Cyclosa sp. 2 & & 1 & 1 \\
\hline$"$ & Cyclosa sp. 3 & 2 & 10 & 12 \\
\hline$"$ & Eustala eleuthera Levi, 1977 & & 1 & 1 \\
\hline$"$ & Eustala sp. 1 & 21 & 22 & 43 \\
\hline
\end{tabular}


CUADRO 1 (Continuación)

Especies y morfoespecies de arañas en cultivos de Citrus sinensis en Bella Vista, Corrientes, Argentina

TABLE 1 (Continued)

Species and morphospecies of spiders in cultures of Citrus sinensis in Bella Vista, Corrientes, Argentina

\begin{tabular}{|c|c|c|c|c|}
\hline Familia & Especies / Morfoespecies & AM1 & AM2 & Total \\
\hline$"$ & Eustala sp. 2 & 3 & & 3 \\
\hline$"$ & Gasterocantha cancriformis (Linnaeus, 1758) & & 1 & 1 \\
\hline$"$ & Mecynogea lemniscata (Walckenaer, 1841) & 1 & & 1 \\
\hline$"$ & Metepeira compsa (Chamberlin, 1916) & 1 & 2 & 3 \\
\hline$"$ & Micrathena sp. 1 & 1 & & 1 \\
\hline ” & Metepeira sp. 1 & 9 & 6 & 15 \\
\hline$"$ & Ocrepeira fiebrigi (Dahl, 1906) & 3 & & 3 \\
\hline$"$ & Ocrepeira venustula (Keyserling, 1879) & 7 & 3 & 10 \\
\hline$"$ & Ocrepeira sp. 1 & 3 & & 3 \\
\hline$"$ & Parawixia sp. 1 & 41 & 29 & 70 \\
\hline$"$ & Parawixia sp. 2 & 8 & 2 & 10 \\
\hline$"$ & Parawixia sp. 3 & 3 & 5 & 8 \\
\hline$"$ & Parawixia sp. 4 & 1 & 5 & 6 \\
\hline$"$ & Parawixia audax (Blackwall, 1863) & 1 & & 1 \\
\hline$"$ & Parawixia bistriata (Rengger, 1836) & 2 & 2 & 4 \\
\hline$"$ & Parawixia monticula (Keyserling, 1892) & & 1 & 1 \\
\hline$"$ & Parawixia velutina (Taczanowski, 1878) & 4 & 10 & 14 \\
\hline$"$ & Pronous sp. 1 & 1 & & 1 \\
\hline$"$ & Scoloderus sp. 1 & 6 & 6 & 12 \\
\hline$"$ & Wagneriana sp. 1 & 1 & & 1 \\
\hline$"$ & Morfo sp. 1 & 5 & & 5 \\
\hline Caponidae & Morfo sp. 1 & 2 & 1 & 3 \\
\hline Corinnidae & Morfo sp. 1 & 23 & 18 & 41 \\
\hline$"$ & $" \quad$ sp. 2 & 94 & 62 & 156 \\
\hline$"$ & $" \quad$ sp. 3 & 6 & 4 & 10 \\
\hline$"$ & $" \quad$ sp. 4 & 73 & 91 & 164 \\
\hline$"$ & ” $\quad$ sp. 5 & 18 & 12 & 30 \\
\hline$"$ & $"$ sp. 6 & 3 & 3 & 6 \\
\hline$"$ & $" \quad$ sp. 7 & 11 & 4 & 15 \\
\hline$"$ & $" \quad$ sp. 8 & 3 & 1 & 4 \\
\hline$"$ & Trachelas sp. 1 & 1 & 2 & 3 \\
\hline$”$ & Trachelopachis sp. 1 & 2 & 2 & 4 \\
\hline Ctenidae & Ancylometes concolor (Perty, 1833) & 2 & 1 & 3 \\
\hline$"$ & Morfo sp. 1 & 4 & 3 & 7 \\
\hline Dictynidae & Morfo sp. 1 & 9 & 20 & 29 \\
\hline$"$ & $"$ sp. 2 & 36 & 26 & 62 \\
\hline$"$ & $"$ sp. 3 & 1 & 1 & 2 \\
\hline Gnaphosiade & Morfo sp. 1 & 57 & 14 & 71 \\
\hline$"$ & $" \quad$ sp. 2 & 6 & 5 & 11 \\
\hline$"$ & $" \quad$ sp. 3 & 16 & 6 & 22 \\
\hline$"$ & $" \quad$ sp. 4 & 16 & 11 & 27 \\
\hline$"$ & $" \quad$ sp. 5 & 75 & 41 & 116 \\
\hline$"$ & $"$ sp. 6 & 4 & & 4 \\
\hline$"$ & $" \quad$ sp. 7 & & 1 & 1 \\
\hline
\end{tabular}


CUADRO 1 (Continuación)

Especies y morfoespecies de arañas en cultivos de Citrus sinensis en Bella Vista, Corrientes, Argentina

TABLE 1 (Continued)

Species and morphospecies of spiders in cultures of Citrus sinensis in Bella Vista, Corrientes, Argentina

\begin{tabular}{|c|c|c|c|c|}
\hline Familia & Especies / Morfoespecies & AM1 & AM2 & Total \\
\hline Hahniidae & $" \quad$ sp. 1 & 9 & 15 & 24 \\
\hline Hersiliidae & Iviraiva pachyura (Mello-Leitão, 1935) & 155 & 86 & 241 \\
\hline Linyphiidae & Morfo sp. 1 & 135 & 164 & 299 \\
\hline ” & $"$ sp. 2 & 7 & 19 & 26 \\
\hline$"$ & ” sp. 3 & 8 & 8 & 16 \\
\hline$"$ & $"$ sp. 4 & 72 & 85 & 157 \\
\hline$"$ & $" \quad$ sp. 5 & 16 & 12 & 28 \\
\hline$"$ & $"$ sp. 6 & 28 & 20 & 48 \\
\hline$"$ & $"$ sp. 7 & 22 & 31 & 53 \\
\hline$"$ & $" \quad$ sp. 8 & 100 & 50 & 150 \\
\hline$"$ & ” $\quad$ sp. 9 & 1 & 6 & 7 \\
\hline$"$ & $"$ sp. 10 & 8 & 16 & 24 \\
\hline$"$ & $" \quad$ sp. 11 & 1 & 2 & 3 \\
\hline$"$ & $" \quad$ sp. 12 & 1 & & 1 \\
\hline Lycosidae & Morfo sp. 1 & 31 & 35 & 66 \\
\hline$"$ & $" \quad$ sp. 2 & 61 & 25 & 86 \\
\hline$"$ & " $\quad$ sp. 3 & 22 & 9 & 31 \\
\hline$"$ & $=\quad$ sp. 4 & 363 & 230 & 593 \\
\hline$"$ & $" \quad$ sp. 5 & 97 & 126 & 223 \\
\hline$"$ & $"$ sp. 6 & 155 & 66 & 221 \\
\hline Mimetidae & Mimetus melanoleucus Mello-Leitão, 1929 & 3 & 2 & 5 \\
\hline$"$ & Mimetus sp. 1 & 4 & 4 & 8 \\
\hline$"$ & Morfo sp. 1 & 5 & 4 & 9 \\
\hline Miturgidae & Cheiracanthium inclusum (Hentz, 1847) & 99 & 141 & 240 \\
\hline$"$ & Teminius agalenoides (Badcock, 1932) & 21 & 20 & 41 \\
\hline Oonopidae & Gamasomorpha patquiana Birabén, 1954 & 6 & 2 & 8 \\
\hline$"$ & Gamasomorpha platensis Birabén, 1954 & 1 & & 1 \\
\hline$"$ & Gamasomorpha sp. & 5 & 1 & 6 \\
\hline$"$ & Oonops nigromaculatus Mello-Leitão, 1944 & 8 & & 8 \\
\hline$"$ & Oonops sp. & 1 & & 1 \\
\hline$"$ & Gamasomorpha wasmanniae Mello-Leitão, 1939 & 2 & & 2 \\
\hline Oxyopidae & Morfo sp. 1 & 5 & 11 & 16 \\
\hline$"$ & $"$ sp. 2 & & 4 & 4 \\
\hline$"$ & $=$ sp. 3 & 9 & 13 & 22 \\
\hline$"$ & "sp. 4 & 3 & 3 & 6 \\
\hline$"$ & $"$ sp. 5 & 2 & 4 & 6 \\
\hline$"$ & $"$ sp. 6 & 2 & 4 & 6 \\
\hline$"$ & $"$ sp. 7 & & 1 & 1 \\
\hline Palpimanidae & Otiothops sp. 1 & 2 & & 2 \\
\hline Philodromidae & Morfo sp. 1 & 4 & 1 & 5 \\
\hline$"$ & " sp. 2 & 6 & 9 & 15 \\
\hline Pholcidae & Morfo sp. 1 & 9 & 6 & 15 \\
\hline Pisauriidae & Morfo sp. 1 & 6 & 1 & 7 \\
\hline
\end{tabular}


CUADRO 1 (Continuación)

Especies y morfoespecies de arañas en cultivos de Citrus sinensis en Bella Vista, Corrientes, Argentina

TABLE 1 (Continued)

Species and morphospecies of spiders in cultures of Citrus sinensis in Bella Vista, Corrientes, Argentina

\begin{tabular}{|c|c|c|c|c|}
\hline Familia & Especies / Morfoespecies & AM1 & AM2 & Total \\
\hline Prodidomidae & Morfo sp. 1 & 71 & 74 & 145 \\
\hline$"$ & $" \quad$ sp. 2 & 3 & 6 & 9 \\
\hline$"$ & $"$ sp. 3 & & 1 & 1 \\
\hline Salticidae & $" \quad$ sp. 1 & 51 & 51 & 102 \\
\hline$"$ & $" \quad$ sp. 2 & 8 & 1 & 9 \\
\hline$"$ & $"$ sp. 3 & 51 & 24 & 75 \\
\hline$"$ & ” $\quad$ sp. 4 & 5 & 4 & 9 \\
\hline$"$ & $"$ sp. 5 & 12 & 16 & 28 \\
\hline$"$ & $"$ sp. 6 & 12 & 10 & 22 \\
\hline$"$ & $"$ sp. 7 & 72 & 24 & 96 \\
\hline$"$ & $" \quad$ sp. 8 & 4 & 2 & 6 \\
\hline$"$ & $" \quad$ sp. 9 & 11 & 13 & 24 \\
\hline$"$ & " sp. 10 & 2 & 3 & 5 \\
\hline$"$ & $" \quad$ sp. 11 & 5 & 4 & 9 \\
\hline$"$ & " $\quad$ sp. 12 & 13 & 1 & 14 \\
\hline$"$ & $" \quad$ sp. 13 & 9 & 19 & 28 \\
\hline$"$ & " sp. 14 & 20 & 20 & 40 \\
\hline$"$ & $" \quad$ sp. 15 & 2 & 2 & 4 \\
\hline$"$ & " $\quad$ sp. 16 & 10 & 18 & 28 \\
\hline$"$ & " $\quad$ sp. 17 & 6 & 11 & 17 \\
\hline$"$ & " sp. 18 & 1 & 4 & 5 \\
\hline$"$ & " $\quad$ sp. 19 & 3 & 5 & 8 \\
\hline$"$ & " $\quad$ sp. 20 & 1 & 2 & 3 \\
\hline$"$ & $=\quad$ sp. 21 & 21 & 24 & 45 \\
\hline$"$ & $" \quad$ sp. 22 & 18 & 14 & 32 \\
\hline$"$ & " $\quad$ sp. 23 & 1 & & 1 \\
\hline$"$ & $" \quad$ sp. 24 & 8 & 20 & 28 \\
\hline$"$ & $" \quad$ sp. 25 & 25 & 32 & 57 \\
\hline$"$ & $" \quad$ sp. 26 & 4 & 2 & 6 \\
\hline$"$ & " $\quad$ sp. 27 & 21 & 10 & 31 \\
\hline$"$ & $=\quad$ sp. 28 & 9 & 15 & 24 \\
\hline$"$ & " $\quad$ sp. 29 & 7 & 15 & 22 \\
\hline$"$ & ” $\quad$ sp. 30 & 4 & 6 & 10 \\
\hline$"$ & " $\quad$ sp. 31 & 4 & 1 & 5 \\
\hline$"$ & " $\quad$ sp. 32 & 10 & 10 & 20 \\
\hline$"$ & " sp. 33 & & 1 & 1 \\
\hline$"$ & $" \quad$ sp. 34 & 1 & 2 & 3 \\
\hline$"$ & " $\quad$ sp. 35 & & 1 & 1 \\
\hline$"$ & $" \quad$ sp. 36 & 1 & 3 & 4 \\
\hline$"$ & " $\quad$ sp. 37 & & 3 & 3 \\
\hline$"$ & " $\quad$ sp. 38 & 6 & 10 & 16 \\
\hline Scytodidae & Scytodes eleonorae Rheims \& Brescovit, 2001 & 3 & & 3 \\
\hline$"$ & Scytodes strussmannae Rheims \& Brescovit, 2001 & 2 & & 2 \\
\hline$"$ & Scytodes tuуисиа Brescovit, Rheims \& Raizer, 2004 & 34 & 80 & 114 \\
\hline
\end{tabular}


CUADRO 1 (Continuación)

Especies y morfoespecies de arañas en cultivos de Citrus sinensis en Bella Vista, Corrientes, Argentina

TABLE 1 (Continued)

Species and morphospecies of spiders in cultures of Citrus sinensis in Bella Vista, Corrientes, Argentina

\begin{tabular}{|c|c|c|c|c|}
\hline Familia & Especies / Morfoespecies & AM1 & AM2 & Total \\
\hline Selenopidae & Selenps sp. 1 & 1 & & 1 \\
\hline Senoculidae & Senoculus sp. 1 & 3 & 7 & 10 \\
\hline Sparassidae & Polybetes rapidus (Keyserling, 1880) & 41 & 22 & 63 \\
\hline Tetragnathidae & Leucauge venusta (Walckenaer, 1841) & 212 & 205 & 417 \\
\hline$"$ & Tetragnatha sp. 1 & 12 & 9 & 21 \\
\hline Titanoecidae & Goeldia sp. 1 & 7 & 4 & 11 \\
\hline Theridiidae & Anelosimus sp. 1 & 27 & 55 & 82 \\
\hline " & Morfo sp. 1 & 2 & 5 & 7 \\
\hline ” & $"$ sp. 2 & 15 & 5 & 20 \\
\hline ” & $"$ sp. 3 & 126 & 147 & 273 \\
\hline$"$ & $" \quad$ sp. 4 & 8 & 5 & 13 \\
\hline$"$ & $" \quad$ sp. 5 & 6 & 4 & 10 \\
\hline$"$ & $" \quad$ sp. 6 & 8 & 10 & 18 \\
\hline$"$ & $" \quad$ sp. 7 & 1 & & 1 \\
\hline$"$ & $" \quad$ sp. 8 & 3 & & 3 \\
\hline$"$ & $" \quad$ sp. 9 & 12 & 16 & 28 \\
\hline$"$ & $" \quad$ sp. 10 & 8 & 15 & 23 \\
\hline$"$ & " sp. 11 & 2 & 2 & 4 \\
\hline$"$ & " sp. 12 & 17 & 3 & 20 \\
\hline$"$ & $" \quad$ sp. 13 & 4 & 2 & 6 \\
\hline$"$ & $" \quad$ sp. 14 & 3 & 1 & 4 \\
\hline$"$ & $" \quad$ sp. 15 & 8 & 10 & 18 \\
\hline ” & $" \quad$ sp. 16 & 1 & 3 & 4 \\
\hline ” & " $\quad$ sp. 17 & & 1 & 1 \\
\hline$"$ & " $\quad$ sp. 18 & 4 & 1 & 5 \\
\hline$"$ & " $\quad$ sp. 19 & & 1 & 1 \\
\hline$"$ & " $\quad$ sp. 20 & 3 & 1 & 4 \\
\hline$"$ & " $\quad$ sp. 21 & & 1 & 1 \\
\hline$"$ & $=\quad$ sp. 22 & & 1 & 1 \\
\hline Thomisidae & Tmarus sp. & 7 & 3 & 10 \\
\hline$"$ & Morfo sp. 1 & 12 & 8 & 20 \\
\hline$"$ & $" \quad$ sp. 2 & 29 & 18 & 47 \\
\hline$"$ & $" \quad$ sp. 3 & 5 & 5 & 10 \\
\hline$"$ & " sp. 4 & 4 & 2 & 6 \\
\hline$"$ & $" \quad$ sp. 5 & 9 & 12 & 21 \\
\hline Trechaleidae & Morfo sp. 1 & 9 & 7 & 16 \\
\hline$"$ & $" \quad$ sp. 2 & 3 & 1 & 4 \\
\hline Uloboridae & Uloborus sp. 1 & 1 & 1 & 2 \\
\hline Zodariidae & Cybaeodamus ornatus Mello-Leitão, 1938 * & 54 & 58 & 112 \\
\hline \multirow[t]{2}{*}{$"$} & Cybaeodamus enigmaticus (Mello-Leitão, 1939) & 4 & 4 & 8 \\
\hline & Total & 3811 & 3363 & 7174 \\
\hline
\end{tabular}



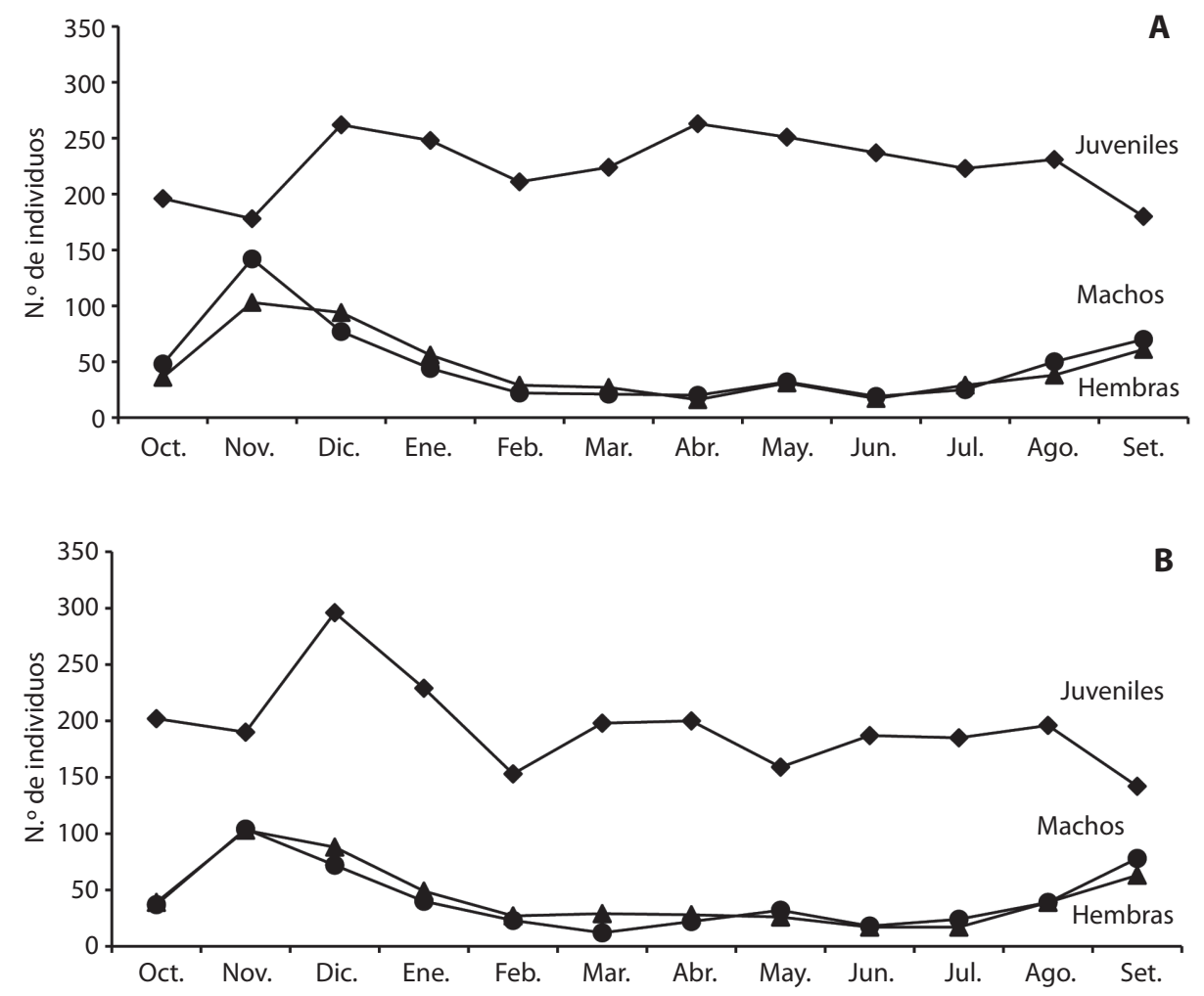

Fig. 1. A. Total de individuos recolectados por mes según sexo y estado de desarrollo en cultivos con riego de Citrus sinensis en Bella Vista, Corrientes, Argentina. B. Total de individuos recolectados por mes según sexo y estado de desarrollo en un cultivo sin riego en Citrus sinensis en Bella Vista, Corrientes, Argentina.

Fig. 1. A. Total of individuals collected per month by sex and stage of development in cultures of Citrus sinensis irrigated in Bella Vista, Corrientes, Argentina. B. Total of individuals collected per month by sex and stage of development in cultures of Citrus sinensis unirrigated in Bella Vista, Corrientes, Argentina.

De las familias determinadas, ocho fueron las más abundantes y representaron el $75 \%$ del total de arañas recolectadas en las dos áreas evaluadas, siendo Lycosidae la más numerosa (Fig. 2). La morfoespecie 4 de la familia Lycosidae fue la más numerosa de las arañas recolectadas $(\mathrm{n}=593)$, seguida por Leucauge venusta (Tetragnathidae) $(\mathrm{n}=417)$, morfoespecie 1 (Linyphiidae) $(\mathrm{n}=299)$, Jessica erythrostoma (Anyphaenidae) $(\mathrm{n}=278)$, morfoespecie 3 (Theridiidae) $(\mathrm{n}=273)$, Araneus uniformis (Araneidae) $(\mathrm{n}=256)$, Iviraiva pachyura $(\mathrm{Her}-$ siliidae) $(\mathrm{n}=241)$, Cheiracanthium inclusum (Miturgidae) $(\mathrm{n}=240)$ y morfoespecies 5 y 6 (Lycosidae) ( $\mathrm{n}=223$ y $\mathrm{n}=221$ respectivamente) (Cuadro 1).
De las especies recolectadas surgen cuatro nuevas citas para la Argentina: Eustala eleuthera Levi, 1977 (Araneidae), Ocrepeira fiebrigi (Dahl 1906) (Araneidae), Parawixia monticola (Keyserling 1892) (Araneidae) y Scytodes tuyucua Rheims \& Raizer 2004 (Scytodidae) y 12 nuevas citas para la Provincia de Corrientes: Jessica fidelis (Mello-Leitão 1922) (Anyphaenidae), Aysha triunfo Brescovit 1992 (Anyphaenidae), Osoriella tahela Brescovit 1998 (Anyphaenidae), Acacesia hamata (Hentz 1847) (Araneidae), Cyclosa diversa (O. P.-Cambridge 1894) (Araneidae), C. fililineata Hingston 1932 (Araneidae), C. serena Levi 1999 (Araneidae), C. tapetifaciens Hingston 1932 (Araneidae), I. pachyura (Mello-Leitão 


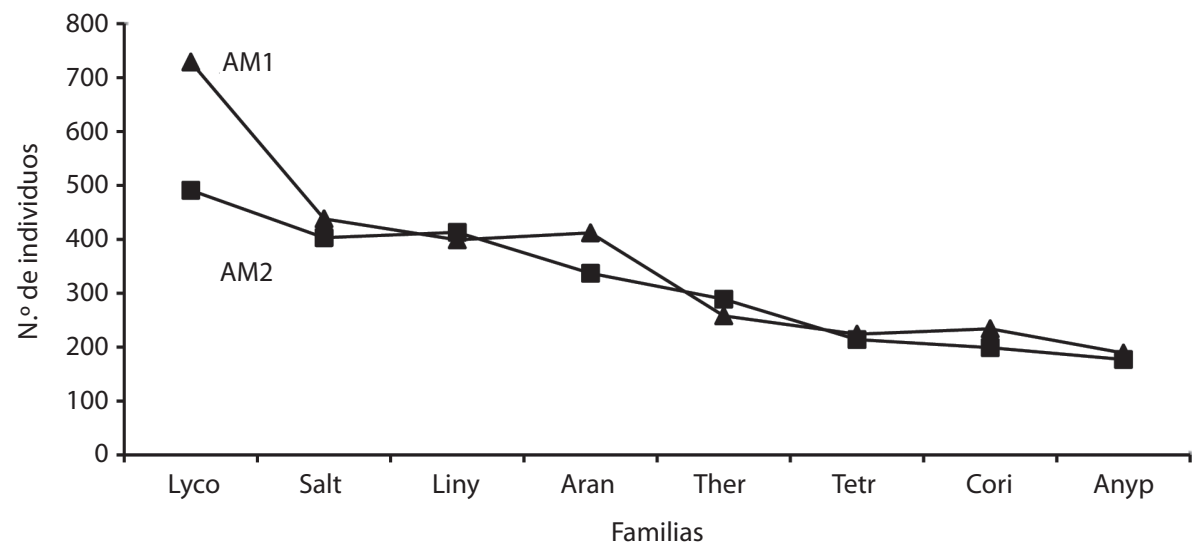

Fig. 2. Abundancia de familias de arañas en cultivos de Citrus sinensis en Bella Vista, Corrientes, Argentina (AM1: con riego, AM2: sin riego). Lyco=Lycosidae, Salt=Salticidae, Liny=Linyphiidae, Aran=Araneidae, Ther=Theridiidae, Tetr=Tetragnathidae, Cori=Corinnidae y Anyp=Anyphaenidae.

Fig. 2. Abundance of spider families in cultures of Citrus sinensis in Bella Vista, Corrientes, Argentina (AM1: irrigated, AM2 unirrigated). Lyco=Lycosidae, Salt=Salticidae, Liny=Linyphiidae, Aran=Araneidae, Ther=Theridiidae, Tetr=Tetragnathidae, Cori=Corinnidae y Anyp=Anyphaenidae.

1935) (Hersiliidae), C. inclusum (Hentz 1847) (Miturgidae), Teminius agalenoides (Badcock 1932) (Miturgidae) y Cybaeodamus enigmaticus (Mello-Leitão 1939) (Zodariidae).

En total se identificaron ochos gremios: constructoras de telas orbiculares, constructoras de telas sábanas, vagabundas de vegetación, vagabundas del suelo, cazadoras al acecho, cazadoras por emboscada, constructoras de telas espaciales y constructoras de telas tubulares. En ambas parcelas, el gremio de las vagabundas de suelo fue el de mayor abundancia (n) y de especies (s) (AM1 n=1341, s=39; AM2 $\mathrm{n}=999, \mathrm{~s}=33$ ), seguido por el de constructoras de telas orbiculares (AM1 n=637, s=36; AM2 $\mathrm{n}=552, \mathrm{~s}=33$ ), las cazadoras al acecho (AM1 $\mathrm{n}=471, \mathrm{~s}=43$; AM2 $\mathrm{n}=453, \mathrm{~s}=47$ ) y por el de telas espaciales (AM1 n=446, s=23; AM2 $\mathrm{n}=342, \mathrm{~s}=25$ ).

En el dendrograma de similitud cuantitativa de AM1 se visualizaron cuatro grandes grupos, que reflejan las cuatro estaciones climáticas del año. En todos los casos con una similitud que oscila entre 66.9 y $54.1 \%$. El mes de octubre se observó como independiente del resto (Fig. 3). Asimismo en AM2 se observaron cuatro grupos, pero con una menor similitud entre los meses respecto a el AM1, ya que los valores oscilaron entre 65.5 y $45 \%$. (Fig. 4).

De acuerdo con el análisis de la diversidad alfa de las arañas recolectadas en las dos unidades de muestreo de $C$. sinensis, no se observó diferencias significativas $(\mathrm{t}=1.083, \mathrm{p}=0.279)$ (Cuadro 2).

Del análisis de las funciones de acumulación de especies se verificó que el modelo que

\section{CUADRO 2}

Diversidad de arañas en cultivos de Citrus sinensis en Bella Vista, Corrientes, Argentina

TABLE 2

Spider diversity in cultures of Citrus sinensis in Bella Vista, Corrientes, Argentina

\begin{tabular}{ccc} 
Índices & AM1 & AM2 \\
S & 179 & 174 \\
N & 3811 & 3363 \\
D & 0.02804 & 0.02582 \\
H' & 4.161 & 4.184 \\
J' & 0.8021 & 0.8111 \\
\hline
\end{tabular}

Shannon-Wiener (H'), dominancia de Simpson (D) y equidad de Pielou (J'), AM1: con riego, AM2: sin riego. Shannon-Wiener $\left(\mathrm{H}^{\prime}\right)$, Simpson dominance (D) and equity of Pielou (J'), AM1: irrigated, AM2 unirrigated. 


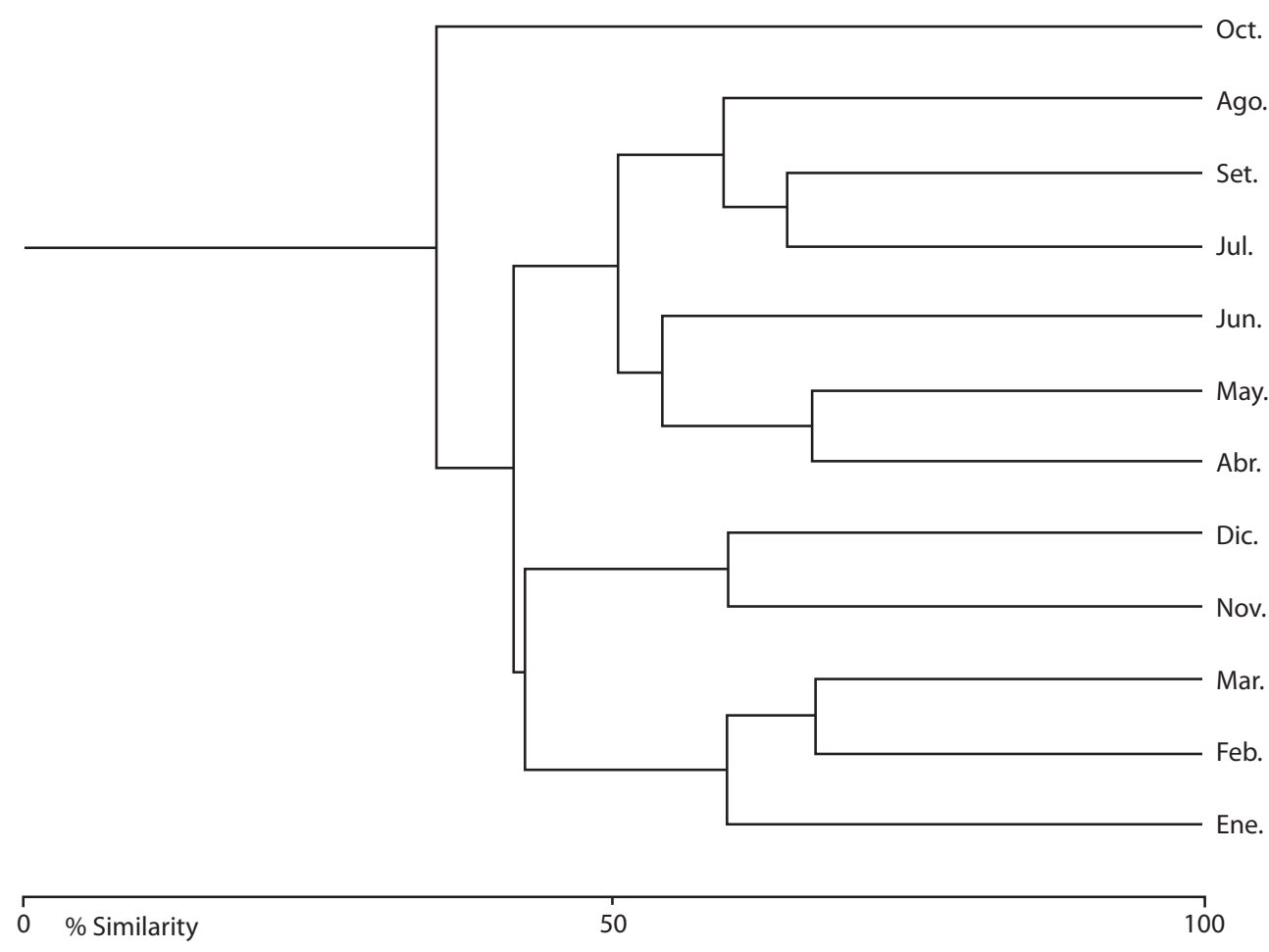

Fig. 3. Dendrograma de similitud del cultivo con riego de Citrus sinensis en Bella Vista, Corrientes, Argentina. Fig. 3. Dendrogram of similarity in cultures of Citrus sinensis irrigated in Bella Vista, Corrientes, Argentina.

mejor ajustó los resultados, fue el de dependencia lineal, donde en general las especies observadas fueron más que las estimadas por el modelo. Asimismo, el modelo de Clench estimó un $90.9 \%$ de las arañas observadas en el AM1 y un $90.6 \%$ en el AM2, lo que demuestra que el esfuerzo de muestreo fue suficiente (Cuadro 3). Además, los resultados obtenidos con otros estimadores muestran resultados similares (Cuadro 4).

\section{DISCUSIÓN}

La araneofauna recolectada representa el $63.5 \%$ de las familias de Araneomorphae identificadas para Argentina, que agrupa actualmente a 52 familias (Platnick 2012), y el $79.5 \%$ de las familias registradas de 19 Departamentos de la Provincia de Corrientes (Avalos et al. 2006).
Por otro lado, el número de familias registrado en esta investigación es elevado con relación a otros cultivos de Citrus estudiados: Van Den Berg et al. (1992) en C. sinensis de Sudáfrica, identificaron 21 familias de arañas, y Benamú (2004) en C. limon de Montevideo, Uruguay, identificaron 18 familias en un campo en abandono, y 23 familias en un cultivo convencional.

En el área de estudio no utilizaron agroquímicos para el control de plagas durante el año de muestreo, hecho que podría explicar la abundancia y riqueza de especies de arañas halladas. En coincidencia, Halaj et al. (2000) sostienen que los sistemas agrícolas, especialmente de cultivos perennes como la naranja (C. sinensis), despliegan una estructura arbórea de dimensiones importantes y son muy estables con una rica entomofauna. Dada la estructura de los árboles y los recursos alimenticios 

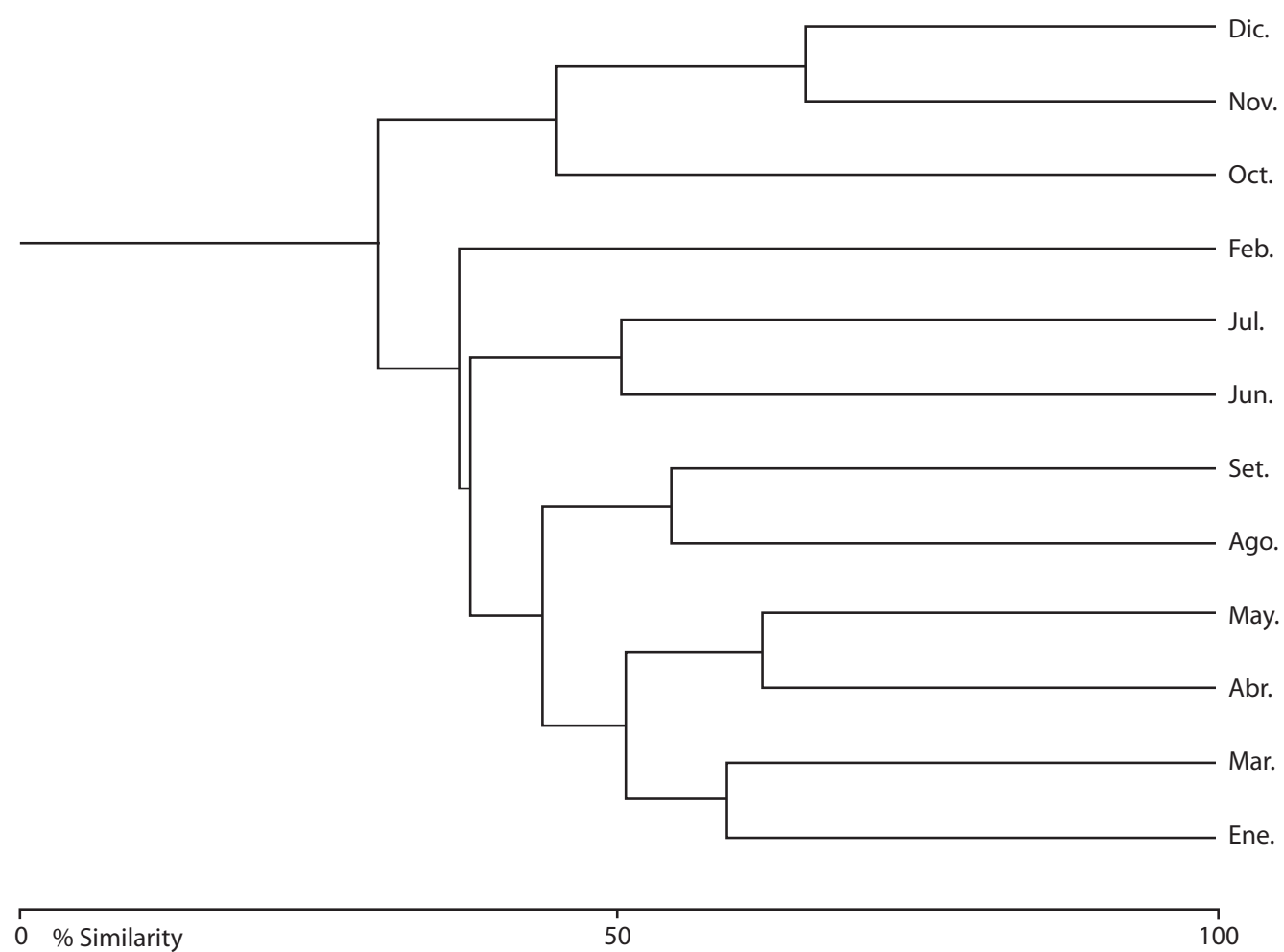

Fig. 4. Dendrograma de similitud del cultivo sin riego de Citrus sinensis en Bella Vista, Corrientes, Argentina.

Fig. 4. Dendrogram of similarity in cultures of Citrus sinensis unirrigated in Bella Vista, Corrientes, Argentina.

\section{CUADRO 3}

Modelos de acumulación de especies de arañas en cultivos de Citrus sinensis en Bella Vista, Corrientes, Argentina

TABLE 3

Models of accumulation of species of spiders in cultured Citrus sinensis in Bella Vista, Corrientes, Argentina

\begin{tabular}{lcccccccc} 
& \multicolumn{3}{c}{ Modelo de dependencia lineal } & \multicolumn{4}{c}{ Modelo de Clench } \\
& A & B & S est & S obs & A & b & S est & S obs \\
Total AM1 & 0.7164 & 0.0042 & 170 & 179 & 1.0831 & 0.0055 & 197 & 179 \\
Total AM2 & 0.6705 & 0.0041 & 164 & 174 & 0.9846 & 0.0051 & 192 & 174 \\
\hline
\end{tabular}

AM1: con riego, AM2: sin riego. / AM1: irrigated, AM2: unirrigated.

presentes, se podría afirmar que la araneofauna que se observó en los cultivos estudiados es semejante a la de los sistemas naturales.

Es importante resaltar la edad de la plantación de los Citrus investigados, con relación a la diversidad de arañas encontradas.
Elizondo-Solís (2002), sostiene que los cambios de la composición de las especies y de la complejidad de la comunidad, sería cada vez mayor con la edad de los ejemplares. Asimismo, Gerez (1988) sostiene que los artrópodos, en general, estarían asociados a una formación 
CUADRO 4

Abundancia (N), riqueza de especies (S) y estimadores de especies de arañas en cultivos de Citrus sinensis en Bella Vista, Corrientes, Argentina

TABLE 4

Abundance (N), species richness (S) and estimates of spider species in cultures of Citrus sinensis in Bella Vista, Corrientes, Argentina

\begin{tabular}{lcccccccc} 
& Muestra & $\mathrm{N}$ & $\mathrm{S}$ & Chao1 & Chao2 & Jack1 & Jack2 \\
AM1 & 1440 & 3811 & 179 & 199.6 & 202.9 & 208 & 221 & 225 \\
AM2 & 1440 & 3363 & 174 & 203.5 & 203.7 & 209 & 232 & 255 \\
\hline AM1 y AM2 & 2880 & 7174 & 200 & 240.6 & 249.6 & 232 & \\
\hline
\end{tabular}

(AM1: con riego, AM2: sin riego) / (AM1: irrigated, AM2: unirrigated).

vegetal, dependiendo del grado de especialización que presentan. Desde esta perspectiva los árboles proveen refugios para la alimentación y oviposición, para el acoplamiento sexual y protección de los efectos climáticos adversos (Lawton 1983).

Se observó un predominio de juveniles durante el año, aumentando levemente su abundancia en verano y otoño. Según Duffey (1962) y Breymeyer (1966), los adultos de Araneomorphae no superan el $48 \%$ de las poblaciones naturales de arañas, coincidiendo con los resultados de esta investigación; en la cual la población adulta alcanzó el $29.1 \%$ en la unidad con riego y el $30.5 \%$ en la unidad sin riego.

La abundancia de adultos en primavera y verano, coincide con las épocas de mayor abundancia de insectos y ácaros que pueden dañar las plantaciones de Citrus (Cáceres 2006).

Asimismo, el mayor número de arañas observado en primavera y principios del verano (noviembre y diciembre), en ambas áreas de muestreos, indicaría que durante estas estaciones climáticas se presentan condiciones ambientales favorables, que le confieren a la araneofauna una mayor capacidad reproductiva. Sin embargo, en enero, cuando la temperatura fue muy elevada la población disminuyó. Real et al. (1999) sostienen que los efectos negativos que se pueden observar en la abundancia de especies, podría deberse no solo a las altas temperaturas, sino también a la transpiración de los vegetales. Estos autores sostienen que cuando estos factores son excesivos para los requerimientos fisiológicos de las especies, pueden provocar un estrés ambiental sobre las mismas.

Las arañas del gremio vagabundas del suelo fueron las que mayor número de individuos aportaron a la abundancia total. Nyffeler $\&$ Sunderland (2003) sugieren que esta particularidad se debe a la amplia dieta observada en estas arañas. Así, en este gremio, se verificaron familias con abundantes individuos, siendo Lycosidae la que prevalece numéricamente dentro del mismo y dentro del total de las familias determinadas en esta investigación.

El uso de unidades taxonómicas reconocibles (morfoespecies) ha demostrado ser útil para una rápida estimación de la biodiversidad (Oliver \& Beattie 1993). Oliver \& Beattie (1996a, 1996b) han encontrado que las estimaciones de la riqueza de hormigas y arañas varían muy poco entre los inventarios de morfoespecies y de especies. En coincidencia, esta herramienta permitió un análisis pormenorizado de la diversidad de arañas en el área de estudio.

El número de especies raras registradas en la plantación de Citrus es bastante elevado, hecho observado en los singletons y doubletons de ambas unidades. En coincidencia, New (1999) sostiene que en los inventarios de arañas, el número de especies raras generalmente es elevado. De hecho, en ninguno de los trabajos en los que se estudia la calidad de los inventarios de arañas se consigue registrar la totalidad de las especies; por ejemplo, los 
inventarios de Coddington et al. (1996) y Sørensen et al. (2002) registran porcentajes de singletons del $29.3 \%$ y $19 \%$, respectivamente.

En general, no existe diferencias significativas en la diversidad alfa entre las dos parcelas, sin embargo, las diferencias que se pueden observar entre ellas se registró en los primeros meses del año, donde fue mayor en el AM1. Este hecho podría deberse a que la estructura y el tamaño de los árboles de la unidad con riego presentan un mayor desarrollo que los de la unidad sin riego. En coincidencia, Uetz (1991) y Benamú (2004), sostienen la existencia de una correlación entre la complejidad estructural del hábitat y la diversidad de especies de arañas. Así, el AM1 ofrece una mayor oferta de refugios y alimentación (mayor desarrollo del follaje y floración, mayor número de insectos) durante todo el año y las fluctuaciones de diversidad observadas no son tan marcadas con relación al AM2, que deben compensar las pérdidas de individuos y especies en el invierno y reponerlas en el verano con mayor eficiencia.

Jiménez-Valderde \& Hortal (2003) sostienen que al menos que se alcance el número asintótico de especies, no existen criterios objetivos que permitan decidir cuándo se considera un inventario lo suficientemente completo. Los investigadores suelen establecer límites arbitrarios para determinar si los muestreos han sido lo suficientemente exhaustivos. En general, para la ecuación de Clench y con el número de individuos o de registros en una base de datos como unidad de esfuerzo, a partir de proporciones superiores al $70 \%$, las estimas de la riqueza asintótica se hacen estables.

La abundancia y diversidad de la fauna de arañas en ambos cultivos demostraría que la utilización de este tipo de manejo (con y sin riego), no afecta la dinámica natural y ya que el impacto negativo sobre el medio es nulo, es compatible con el BPA (Buenas Prácticas Agrícolas).

Teniendo en cuenta que los hábitos alimenticios de las arañas se centran principalmente en los insectos y que numéricamente, tanto de especies como de individuos, las arañas superan a la mayoría de los animales cazadores terrestres, se podría decir que éstas son los depredadores más abundantes encontrados en cultivos de Citrus, por lo que es fundamental resaltar su importancia.

Por otra parte, Churchill (1997) estudió los atributos de las arañas como indicadores de cambios ecológicos entre una serie de táxones seleccionados. Este autor concluyó en que las arañas son ideales para estos estudios y merecen ser consideradas para este tipo de evaluación, dado que cumple con una serie de requisitos fundamentales para ser tenidos en cuenta como taxón indicador: son diversas y abundantes, fáciles de muestrear, funcionalmente significativas e interactúan con su ambiente en una manera que pueden reflejar aspectos de cambios ecológicos.

\section{AGRADECIMIENTOS}

Los autores manifiestan su agradecimiento al director del INTA Bella Vista Mario Pedro Lenscak y a Sara Cáceres por su disposición y por brindarnos todo el apoyo necesario para realizar la investigación en el establecimiento. A Mario Ibarra Polesel, María C. Álvarez Bohle, Martina E. Pocco y María E. Prieto por su valiosa colaboración en los muestreos y en la depuración del material.

\section{RESUMEN}

Las arañas son ideales como indicadores de cambios ecológicos por su diversidad y abundancia. En la provincia de Corrientes, Argentina, se estudiaron arañas de Citrus sinensis en parcelas de 0.82 hectáreas, con riego (AM1) y sin riego (AM2). Mediante pitfall, agitación de follaje, captura manual y tamizado de hojarasca se recolectaron 7174 ejemplares (33 familias, 44 géneros y 200 especies/ morfoespecies), el $70 \%$ fueron juveniles. AM1 registró 3811 individuos (33 familias, 179 especies/morfoespecies) y AM2 3363 (31 familias, 174 especies/morfoespecies). Durante el verano se observó la mayor abundancia. En ambas parcelas, ocho familias representaron el $75 \%$ del total, Lycosidae fue la más numerosa y se identificaron ocho gremios; las vagabundas de suelo fueron las más abundantes (AM1 n=1341, s=39; AM2 n=999, s=33) seguida por las constructoras de telas orbiculares (AM1 $n=637$, $\mathrm{s}=36$; AM2 $\mathrm{n}=552, \mathrm{~s}=33$ ), las cazadoras al acecho (AM1 $\mathrm{n}=471, \mathrm{~s}=43$; AM2 $\mathrm{n}=453$, $\mathrm{s}=47$ ) y las de telas espaciales (AM1 $\mathrm{n}=446, \mathrm{~s}=23$; AM2 $\mathrm{n}=342, \mathrm{~s}=25$. La diversidad 
alfa en ambas parcelas (AM1 $\mathrm{H}^{\prime}=4.161, \mathrm{~J}^{\prime}=0.802$; AM2 $\left.\mathrm{H}^{\prime}=4.184, \mathrm{~J}^{\prime}=0.811\right)$ no mostró diferencias significativas $(\mathrm{t}=1.083, \mathrm{p}=0.279)$. El modelo de dependencia lineal fue el que mejor ajustó los resultados. El modelo de Clench estimó el $90.9 \%$ de las observadas en AM1 y el $90.6 \%$ en la AM2.

Palabras clave: Araneae, diversidad, agroecosistemas, enemigos naturales, cítricos.

\section{REFERENCIAS}

Almada, M.S., M.A. Sosa \& A. González. 2012. Araneofauna (Arachnida: Araneae) en cultivos de algodón (Gossypium hirsutum) transgénicos y convencionales en el Norte de Santa Fe, Argentina. Rev. Biol. Trop. 60: 611-623.

Armendano, A. \& A. González. 2010. Estudio de la comunidad de arañas (Arachnida, Araneae) del cultivo de alfalfa en la provincia de Buenos Aires, Argentina. Rev. Biol. Trop. 58: 747-757.

Armendano, A. \& A. González. 2011. Spider fauna associated with wheat crops and adjacent habitats in Buenos Aires, Argentina. Rev. Mex. Biodiv. 82: 1176-1182.

Avalos, G., G.D. Rubio, M.E. Bar \& M.P. Damborsky. 2006. Lista preliminar de la araneofauna (Arachnida: Araneae) del Centro-Norte de la Provincia de Corrientes, Argentina. Rev. Ibérica Aracnol. 13: 189-194.

Bale, J., J. Van Lenteren \& F. Bigler. 2008. Biological control and sustainable food production. 2008. Phil. Trans. R. Soc. B. 363: 761-776.

Beltramo, J., I. Berolaccini \& A. González. 2006. Spiders of soybean crops in Santa Fe Province, Argentina: Influence of surrounding spontaneus vegetation on lot colonization. Braz. J. Biol. 66: 29-41.

Benamú, M. 1999. Estudio preliminar de la araneofauna presente en mandarina cultivada en Vitarte, Lima, Perú. Rev. Per. Entomol. 41: 154-157.

Benamú, M.A. 2004. Estudio Comparativo de la diversidad de arañas de un campo en abandono y un cultivo convencional de limonero (Citrus limon L. Burm) en Rincón del Cerro, Montevideo, Uruguay. Tesis de Maestría, Universidad de Montevideo, Montevideo, Uruguay.

Benamú, M. \& P. Aguilar. 2001. Araneofauna presente en huertos de manzano del valle de mala, Lima, Perú. Rev. Per. Entomol. 42: 199-210.

Birabén, M. 1954. Nuevas Gamasomorphinae de la Argentina (Araneae, Oonopidae). Notas Mus. La Plata 17: 181-212.

Bonaldo, A.B. \& A.D. Brescovit. 1992. As aranhas do Gênero Cheiracanthium C.L. Koch, 1839 na Região Neotropical (Araneae, Clubionidae). Rev. Bras. Entomol. 36: 731-740.
Breene, R.G., D.A. Dean, M. Nyffeler \& G.B. Edwards. 1993. Biology, predation ecology, and significance of spiders in Texas cotton ecosystems with a key to the species. Texas Agric. Exp. Stn. Bull. 1711: 1-115.

Brescovit, A.D. 1996. Revisão de Anyphaeninae Bertkau a nível de Gêneros na Região Neotropical (Araneae, Anyphaenidae). Rev. Bras. Zool. 13: 1-187.

Brescovit, A.D. 1998. Sobre o Gênero Osoriella: Descrição de duas Espécies novas e da Fêmea de $O$. rubella (Araneae, Anyphaenidae, Anyphaeninae). Iheringia Ser. Zool. 84: 109-120.

Brescovit, A.D. 1999. Revisão das aranhas do gêneri Jessica Brescovit (Araneae, Anyphaenidae, Anyphaeninae). Rev. Bras. Entomol. 43: 249-269.

Brescovit, A.D., C.A. Rheims \& J. Raizer. 2004. Notes on the Genus Scytodes Latreille (Araneae, Scytodidae) From the Pantanal, Mato Grosso do Sul, Brazil. Rev. Ibérica Aracnol. 9: 75-79.

Breymeyer, A. 1966. Relations between wandering spiders y other epigeic predatory Arthropoda. Ekol. Pol. 14: $27-7$.

Cabrera, A.L. \& A. Willink. 1973. Biogeografía de América Latina. Monografía 13, Serie de Biología. Organización de Estados Americanos (OEA), Washington, D.C., EE.UU.

Cáceres, S. 2006. Guía Práctica para la Identificación y el Manejo de las Plagas de Citrus. Programa de Reposicionamiento de la Citricultura Correntina, EEA INTA Bella Vista, Corrientes, Argentina.

Churchill, T. 1997. Spiders as ecological indicators: an overview for Australia. Mem. Mus. Victoria 56: 331-337.

Coddington, J.A., L.H. Young \& F.A. Coyle 1996. Estimating spider species richness in a southern Appalachian cove hardwood forest. J. Arachnol. 24: 111-128.

Colwell, R.K. 2010. EstimateS: Statistical estimation of species richness and shared species from samples. Version 8.2. University of Connecticut, Connecticut, EE.UU (Disponible en línea: http://viceroy.eeb. uconn.edu/EstimateS).

Corronca, J.A. 1998. Revisión del género Selenops Latrei1le (Araneae, Selenopidae), para la República Argentina. Acta Zool. Lilloana 44: 313-325.

Dean, D.A. \& W.L. Sterling. 1987. Distribution and abundance patterns of spiders inhabiting cotton in Texas. Texas Agric. Exp. Stn. Bull. 1566: 1-8.

Dondale, C. 1966. The spider fauna (Araneida) of deciduous orchards in the Australian capital territory. Aus. J. Zool. 14: 1157-1192.

Duffey, E. 1962. A population study of spiders in limestone grassland. J. Anim. Ecol. 31: 571-599.

Elizondo-Solís, J.M. 2002. Inventario y fluctuación poblacional de insectos y arañas asociadas con Citrus 
sinensis en la región Huetar Norte de Costa Rica. Manejo Int. Plagas Agroecol. 64: 88-98.

Flórez, E. 1999. Estructura y composición de una comunidad de arañas (Araneae) en un bosque muy seco tropical de Colombia. Bol. Entomol. Venez. 14: 37-51.

Gerez, A.A. 1988. Procalus lenzi y Procalus malaisei (Coleoptera: Chrysomelidae): Dos especialistas del matorral. Rev. Chilena Entomol. 16: 65-67.

Gerschman de Pikelin, B.S. \& R.D. Schiapelli. 1965. El género Polybetes Simon, 1897, en la Argentina. (Araneae, Sparassidae). Rev. Mus. Argent. Cienc. Nat. Entomol. 1: 312-348.

Giménez, L.I., M.C. Goldfarb \& J.F. Casco. 2001. Comportamiento regional de precipitaciones mensuales en la Provincia de Corrientes. Instituto Nacional de Tecnología Agropecuaria EEA, Comunicaciones Científicas y Tecnológicas, Universidad Nacional del Nordeste, Corrientes, Argentina.

Glueck, S. 1994. A taxonomic revision of the orb weaver genus Acacesia (Araneae: Araneidae). Psyche 101: 59-84.

Good, P. 2000. Permutation Tests. A practical guide to resampling methods for testing hypotheses. Springer, Nueva York, EE.UU.

Greenstone, M.H. 1999. Spider predation: how and why we study it. J. Arachnol. 27: 333-342.

Greenstone, M.H. \& A.F. Bennett. 1980. Foraging strategy and metabolic rate in spiders. Ecology 81: 1255-1259.

Grismado, C.J. 2001. Notas sobre ulobóridos de Argentina, con la descripción de una nueva especie del género Uloborus Latrelle de Catamarca (Arachnida, Araneae, Uloboridae). Rev. Mus. Argent. Cienc. Nat. 3: 165-168.

Grismado, C.J. \& M.J. Ramírez. 2002. Descripción de una nueva especie de Otiothops Mac Leay de la Argentina, y notas sobre el género (Araneae, Palpimanidae, Otiothopinae). Rev. Mus. Argent. Cienc. Nat. 4: 99-103.

Grismado, C.J., G. Avalos \& A. González. 2009. First description of the male of Oonops nigromaculatus Mello-Leitão, 1944 (Araneae: Oonopidae) with the redescription of the female and new records. Rev. Mus. Argent. Cienc. Nat. 11: 215-219.

Griswold, C.E., M.J. Ramírez, J.A. Coddington \& N.I. Platnick. 2005. Atlas of phylogenetic data for entelegyne spiders (Araneae, Araneomorphae, Entelegynae), with comments on their phylogeny. Proc. CA Acad. Sci. 56: 1-324.

Halaj, J., D.W. Ross \& A.R. Moldenke. 2000. Importance of habitat structure to the arthropod food-web in Douglas-fir canopies. Oikos 90: 139-152.
Hammer, Ø., D.A.T. Harper \& P.D. Ryan. 2001. PAST: Paleontological Statistics Software package for education and data analysis. Paleontologia Electronica 4: 9. (También disponible en línea: http://palaeoelectronica.org/2001_1/ past issue1_01.htm).

Höfer, H. \& A.D. Brescovit. 2000. A revision of the Neotropical spider genus Ancylometes Bertkau (Araneae: Pisauridae). Insect Syst. Evol. 31: 323-360.

Jackson, R.R. 2000. Prey preferences and visual discrimination ability of Brettus, Cocalus and Cyrba, araneophagic jumping spiders (Araneae, Salticidae) from Australia, Kenya and Sri Lanka. New Zealand. J. Zool. 27: 29-39.

Jiménez-Valderde, A. \& J. Hortal. 2003. Las curvas de acumulación de especies y la necesidad de evaluar la calidad de los inventarios biológicos. Rev. Ibérica Aracnol. 8: 151-161.

Jocqué, R. 1991. A Generic Revision of the Spider Family Zodariidae (Araneae). Bull. Am. Mus. Nat. Hist. 201: 160 .

Krell, F.T. 2004. Parataxonomy versus taxonomy in biodiversity studies-pitfalls and applicability of 'morphospecies' sorting. Entomology, Strength in Diversity, XXII International Congress of Entomology, Brisbane, Queensland, Australia.

Lawton, J.H. 1983. Plant architecture and the diversity of phytophagous insects. Ann. Rev. Entomol. 28: 23-29.

Levi, H.W. 1977. The American Orb-Weaver Genera Cyclosa, Metazygia and Eustala North of Mexico (Araneae, Araneidae). Bull. Mus. Comp. Zool. 148: 61-127.

Levi, H.W. 1980. The Orb-Weaver genus Mecynogea, The Subfamily Metinae and the Genera Pachygnatha, Glenognatha and Azilia of the Subfamily Tetragnathinae north of Mexico (Araneae: Araneidae). Bull. Mus. Comp. Zool. 149: 1-74.

Levi, H.W. 1985. The Spiny Orb-Weaver Genera Micrathe$n a$ and Chaetacis (Araneae: Araneidae). Bull. Mus. Comp. Zool. 150: 429-618.

Levi, H.W. 1988. Orb-Weaving Spiders of de Genus Alpaida (Araneae: Araneidae). Bull. Mus. Comp. Zool. 151: 365-487.

Levi, H.W. 1991a. The American species of the OrbWeaver Genus Carepalxis and new Genus Rubrepeira (Araneae: Araneidae). Psyche 98: 251-264.

Levi, H.W. 1991b. The Neotropical and Mexican Species of the Orb-Weaver Genera Araneus, Dubiepeira and Aculepeira (Araneae: Araneidae). Bull. Mus. Comp. Zool. 152: 163-315.

Levi, H.W. 1991c. The Neotropical Orb-Weaver Genera Edricus and Wagneriana (Araneae: Araneidae). Bull. Mus. Comp. Zool. 152: 359-415. 
Levi, H.W. 1992. Spiders of the Orb-Weaver Genus Prawixia in America (Araneae: Araneidae). Bull. Mus. Comp. Zool. 153: 1-46.

Levi, H.W. 1993. The Neotropical Orb-Weaving Spiders of the Genera Wixia, Pozonia and Ocrepeira (Araneae: Araneidae). Bull. Mus. Comp. Zool. 153: 47-141.

Levi, H.W. 1995. Orb-Weaving Spiders Actinosoma, Spilasma, Micrepeira, Pronous, and Four New Genera (Araneae: Araneidae). Bull. Mus. Comp. Zool. 154: 153-213.

Levi, H.W. 1996. The American Orb-Weavers Hypognatha, Encyosaccus, Xylethrus, Gasteracantha and Enacrosoma (Araneae: Araneidae). Bull. Mus. Comp. Zool. 155: 89-157.

Levi, H.W. 1997. The American Orb-Weaver Genera Mecynogea, Manogea, Kapogea and Cyrtophora (Araneae: Araneidae). Bull. Mus. Comp. Zool. 155: 215-255.

Levi, H.W. 1999. The Neotropical and Mexican OrbWeavers of the Genera Cyclosa and Allocyclosa. (Araneae: Araneidae). Bull. Mus. Comp. Zool. 155: 299-379.

Levi, H.W. 2001. The Systematics of Neotropical OrbWeaving Spiders in the Genus Metepeira (Araneae: Araneidae). Bull. Mus. Comp. Zool. 157: 1-92.

Levi, H.W. 2004. Comments and New Records for the American Genera Gea and Argiope with the description of New Species (Araneae: Araneidae). Bull. Mus. Comp. Zool. 158: 47-66.

Liljesthröm, G., E. Minervino, D. Castro \& A. González. 2002. La comunidad de arañas del cultivo de soja en la provincia de Buenos Aires, Argentina. Neotrop. Entomol. 31: 197-209.

Maloney, D., F. Drummond \& R. Alford. 2003. Spider predation in agroecosystems: can spiders effectively control pest populations? Maine agricultural and forest experiment station Tech. Bull. 190. University of Maine, Maine, EE.UU.

Mansour, F. \& W.H. Whitcomb. 1986. The spiders of a Citrus grove in Israel and their role as biocontrol agents of Ceroplastes floridensis (Homoptera: Coccidae). Entomophaga 31: 269-276.

Mansour, F., J. Ross, G. Edwards, W. Whitcomb \& D. Richman. 1982. Spiders of Florida Citrus groves. Florida Entomol. 65: 514-522.

McAleece, N., P. Lambshead, G. Paterson \& J. Cage. 1997. Biodiversity Professional (V.2.0). The Natural History Museum and The Scottish Association for Marine Science, Oban, Escocia. (También disponible en línea: http://www.sams.ac.uk/dml/projects/ benthic/bdpro/index. htm).

Mello Leitão, C.F. 1929. Mimetideos do Brasil. Rev. Mus. Paulista 16: 537-568.
Ministerio de Producción, Trabajo y Turismo de Corrientes. 2011. Buenas prácticas agrícolas. Ministerio de Producción, Trabajo y Turismo de Corrientes, Corrientes, Argentina. (Consultado: 06 diciembre 2011, http://www.corrientes.gov.ar).

Moreno, C.E. 2001. Manual de métodos para medir la biodiversidad. Universidad Veracruzana, Xalapa, México.

Morrone, J.J. 2001. Biogeografía de América Latina y el Caribe. M\&T-Manuales \& Tesis SEA, Zaragoza, España.

Nentwig, W. 1986. The Prey of Spiders, p. 249-263. In W. Nentwig (ed.). Ecophysiology of Spiders. Springer, Berlin-Heidelberg, Alemania.

New, T.R. 1999. Untangling the web: spiders and the challenges of invertebrate conservation. J. Insect Conserv. 3: 251-256.

Nyffeler, M. \& K. Sunderland. 2003. Composition, abundance and pest control potential of spider communities in agroecosystems: a comparison of European and US studies. Agro. Ecosyst. Environ. 95: 579-612.

Nyffeler, M., D.A. Dean \& L. Sterling. 1992. Diets, feeding specialization, and predatory role of two lynx spiders, Oxyopes salticus and Peucetia viridans (Araneae, Oxyopidae) in Texas cotton agroecosystem. Environ. Entomol. 21: 1457-1465.

Nyffeler, M., W. Sterling \& D. Dean. 1994. Insectivorus activities of spiders in United States field crops. J. Appl. Ent. 118: 113-128.

Oliver, I. \& J.A. Beattie. 1993. A possible method for the rapid assessment of biodiversity. Conserv. Biol. 7: 562-568.

Oliver, I. \& J.A. Beattie. 1996a. Invertebrate morphospecies as surrogates for species: a case study. Conserv. Biol. 10: 99-109.

Oliver, I. \& J.A. Beattie. 1996b. Designing a cost-effective invertebrate survey: a test of methods for rapid assessment of biodiversity. Ecol. App. 6: 594-607.

Platnick, N.I. 2012. The world spider catalog, Versión 12.5. American Museum of Natural History, Nueva York, EE.UU. (Consultado: 29 marzo 2012, http://research. amnh.org/iz/spiders/catalog/INTRO1.html).

Ramírez, M.J. 2003. The spider subfamily Amaurobioidinae (Araneae, Anyphaenidae): a phylogenetic revision at the generic level. Bull. Am. Mus. Nat. Hist. 277: 1-262.

Real, R., J. Olivero, J.C. Guerrero, J.M. Vargas \& A.L. Márquez. 1999. Contrastación de hipótesis explicativas de la distribución de la diversidad específica de arañas (Arachida, Araneae en las Islas Canarias). Bol. S.E.A. 26: 573-581.

Rheims, C.A. \& A.D. Brescovit. 2001. New species and records of Scytodes Latreille, 1804 of the "globula 
group" from Brazil (Araneae, Scytodidae). Andrias 15: 91-98.

Rheims, C.A. \& A.D. Brescovit. 2004. Revisióm and cladistic anáysis of the spider family Hersiliidae (Arachnida, Araneae) with emphasis on Neotropical and Nearctic species. Insect Syst. Evol. 35: 190-239.

Riechert, S.E. \& T. Lockley. 1984. Spiders as biological control agents. Ann. Rev. Entomol. 29: 299-320.

Rodrigues, E.N.L., M.S. Mendonça Jr. \& R. Ott. 2008. Fauna de aranhas (Arachnida, Araneae) em diferentes estágios do cultivo do arroz irrigado em Cachoeirinha, RS, Brasil. Iheringia Sér. Zool. 98: 362-371.

SAGPyA. 2011. Boletin de Difusion, Manejo Integrado de Plagas en el Sector Agroalimentario. Secretaría de Agricultura, Ganadería, Pesca y Alimentos de la Nación, Buenos Aires, Argentina (Disponible en línea: www.sagpya.mecon.gov.ar).

Sørensen, L.L., J.A. Coddington \& N. Scharff. 2002. Inventorying and estimating subcanopy spider diversity using semiquantitative sampling methods in an Afromontane forest. Environ. Entomol. 31: 319-330.

Sunderland, K. 1999. Mechanisms underlying the effects of spiders on pest populations. J. Arachnol. 27: 308-316.
Symondson, W., K. Sunderland \& M. Greenstone. 2002. Can generalist predators be effective biocontrol agents. Ann. Rev. Entomol. 47: 561-594.

Traw, M.B. 1995. A revision of the Neotropical orb-weaving spider genus Scoloderus (Araneae: Araneidae). Psyche 102: 49-72.

Uetz, G.W. 1991. Habitat structure and spider foraging, p. 325-348. In S.S. Bell, E.D. McCoy \& H.R. Mushinsky (eds.). Habitat Structure: The physical arrangement of objects in space. Chapman \& Hall, Londres, Inglaterra.

Uetz, G.W., J. Halaj \& A.B. Cady. 1999. Pitfal traping in ecological studies of wandering spider. J. Arachnol. 27: 270-280.

Van Den Berg, A.M., A.S. Dippenaar-Schoeman, V.E. Deacon \& S.H. Anderson. 1992. Interactions Between Citrus Psylla, Trioza erytreae (Hem. Triozidae), and spiders in an unsprayed Citrus orchard in the Transvaal Lowveld. Entomophaga 37: 599-608.

Wise, D.H. 1993. Spiders in Ecological Webs. Cambridge University, Cambridge, Inglaterra.

Young, O.P. \& G.B. Edwards. 1990. Spiders in United States field crops and their potential affect on crop pests. J. Arachnol. 18: 1-29. 